Bull. Korean Math. Soc. 51 (2014), No. 4, pp. 1229-1240

http://dx.doi.org/10.4134/BKMS.2014.51.4.1229

\title{
LARGE SCHRÖDER PATHS BY TYPES AND SYMMETRIC FUNCTIONS
}

\author{
Su Hyung An, Sen-Peng Eu, and Sangwook Kim
}

\begin{abstract}
In this paper we provide three results involving large Schröder paths. First, we enumerate the number of large Schröder paths by type. Second, we prove that these numbers are the coefficients of a certain symmetric function defined on the staircase skew shape when expanded in elementary symmetric functions. Finally we define a symmetric function on a Fuss path associated with its low valleys and prove that when expanded in elementary symmetric functions the indices are running over the types of all Schröder paths. These results extend their counterparts of Kreweras and Armstrong-Eu on Dyck paths respectively.
\end{abstract}

\section{Introduction}

A large Schröder path of length $n$ is a lattice path from $(0,0)$ to $(n, n)$ using north steps $N=(0,1)$, diagonal steps $D=(1,1)$, and east steps $E=(1,0)$ such that it does not rise above the diagonal $y=x$. For example, the path $E E D D N E N N D E N$ is a large Schröder path of length 7 . We denote the set of large Schröder paths of length $n$ by $\mathcal{R}_{n}$ and call $r_{n}:=\left|\mathcal{R}_{n}\right|$ a large Schröder number. It is known [13] that the $n$-th large Schröder number is given by $r_{n}=\frac{1}{n} \sum_{k=0}^{n}\left(\begin{array}{c}n \\ k-1\end{array}\right)\left(\begin{array}{l}n \\ k\end{array}\right) 2^{k}$ with $r_{0}=1$. The initial terms are $\left\{r_{n}\right\}_{n \geq 0}=$ $\{1,2,6,22,90,394, \ldots\}_{n \geq 0}$. For simplicity, we will often omit "large" for the rest of the paper.

A Dyck path of length $n$ is a lattice path from $(0,0)$ to $(n, n)$ using north steps $N$ and east steps $E$ such that it does not rise above the diagonal $y=x$. We denote the set of Dyck paths of length $n$ by $\mathcal{D}_{n}$. It is well known [13] that the cardinality $\left|\mathcal{D}_{n}\right|$ is the Catalan number $c_{n}:=\frac{1}{n+1}\left(\begin{array}{c}2 n \\ n\end{array}\right)$, with initial terms $\left\{c_{n}\right\}_{n \geq 0}=\{1,1,2,5,14,42,132, \ldots\}_{n \geq 0}$.

The Dyck paths and the large Schröder paths are closely related by definition and both are fundamental families of lattice paths. Hence it is natural to extend results on Dyck paths to large Schröder paths (or vice versa). In literature there have been several works along this line. For example, there is a notion

Received November 19, 2013.

2010 Mathematics Subject Classification. Primary 05A15, 05 E05.

Key words and phrases. Schröder paths, partial horizontal strips, sparse noncrossing partitions, elementary symmetric functions. 
of $q, t$-Schröder numbers parallel to that of the $q, t$-Catalan numbers $[6,7]$. A bijection between Dyck paths and the facets of cluster complex of type $A$ can be generalized to a bijection between Schröder paths and the faces of generalized complex [5]. The interested readers are referred to $[2,4,14]$ for more examples.

In this paper we obtain three new results on large Schröder paths, extending their counterpart results on Dyck paths.

\subsection{Enumeration by type}

Given an integer partition $\lambda$, let $|\lambda|$ denote the sum of the parts and $\ell(\lambda)$ denote its number of nonzero parts. Also we set $m_{\lambda}:=m_{1}(\lambda) ! m_{2}(\lambda) ! m_{3}(\lambda) ! \cdots$, where $m_{i}(\lambda)$ is the number of parts of $\lambda$ equal to $i$. Note that $m_{\lambda}$ here is not the monomial symmetric function.

For a Dyck path, its type is the integer partition formed by the length of the adjacent east steps. For example, the Dyck path EENENNEEENEENNNN has type $\lambda=(3,2,2,1)$. The enumeration of Dyck paths counted by type is done by Kreweras:

Theorem 1.1 (Kreweras [9]). The number of Dyck paths of length $n$ with type $\lambda$ is

$$
\frac{n(n-1) \cdots(n-\ell(\lambda)+2)}{m_{\lambda}} .
$$

Similarly, for a large Schröder path, its type is the integer partition formed by the number of the adjacent east steps. For example, the large Schröder path $E E N D E N N E E N N D E N$ has type $\lambda=(2,2,1,1)$. Our first main result is to count the number of large Schröder paths by type:

Theorem 1.2 (Theorem 3.2). The number $S c h(n, \lambda)$ of large Schröder paths of length $n$ with type $\lambda$ is

$$
\operatorname{Sch}(n, \lambda)=\left(\begin{array}{c}
n+1 \\
|\lambda|+1
\end{array}\right) \frac{n(n-1) \cdots(n-\ell(\lambda)+2)}{m_{\lambda}} .
$$

\subsection{Partial horizontal strip}

Let $\mu=\left(\mu_{1}, \mu_{2}, \ldots\right)$ and $\nu=\left(\nu_{1}, \nu_{2}, \ldots\right)$ be integer partitions such that $\mu_{i} \geq$ $\nu_{i}$ for all $i$. The skew shape $\mu / \nu$ is the setwise difference of Young diagrams. A partial horizontal strip in the shape $\mu / \nu$ is a set of boxes, at most one in each column, such that the height of the boxes is weakly increasing to the right. The collection of numbers of boxes in each row of a partial horizontal strip $\sigma$ form a partition, called the type of $\sigma$. By an abuse of notation, we will also denote this type by $\sigma$ if there is no confusion. A partial horizontal strip is right-aligned if the result of adding a box to the right of any given box is not a partial horizontal strip.

For a partition $\lambda$, let $h_{\lambda}$ be the homogeneous symmetric function of type $\lambda$ on variables $x_{1}, \ldots, x_{k}$. Let $\lambda \vdash \leq n$ mean that $\lambda \vdash n^{\prime}$ for some $0 \leq n^{\prime} \leq n$. 
It turns out that when $\mu / \nu=n^{n} /(n-1, \ldots, 2,1)$ is a staircase shape, the sum of all homogeneous symmetric functions indexed by right aligned partial horizontal strips has coefficients related to Theorem 1.1:

Theorem 1.3 (Armstrong and $\mathrm{Eu}[1]$ ). We have

$$
\sum_{\sigma} h_{\sigma}=\sum_{\lambda \vdash \leq n} \frac{n !(n+1-|\lambda|)}{m_{\lambda}(n+1-\ell(\lambda))} h_{\lambda},
$$

where the sum on the left hand side is over all right aligned partial horizontal strips $\sigma$ contained in the staircase skew shape $n^{n} /(n-1, \ldots, 2,1)$.

The top homogeneous part of $\sum_{\sigma} h_{\sigma}$ is the parking function symmetric function, introduced by Haiman [8]. The coefficient of $h_{\lambda}$ in the expansion of this function in homogeneous symmetric function bases is the number of Dyck paths of type $\lambda[12]$.

Let $e_{\lambda}$ be the elementary symmetric function of type $\lambda$. Our second main result is the Schröder version of the above. This can be considered as an elementary symmetric function analogue of parking function symmetric functions. In this version the partial horizontal strips need not to be right aligned and the summands are elementary symmetric functions.

Theorem 1.4 (Theorem 4.2). We have

$$
\sum_{\sigma} e_{\sigma}=\sum_{\lambda \vdash n}\left(\begin{array}{c}
n+1 \\
|\lambda|+1
\end{array}\right) \frac{n(n-1) \cdots(n-\ell+2)}{m_{\lambda}} e_{\lambda},
$$

where the sum on the left is over all partial horizontal strips $\sigma$ contained in the staircase skew shape $n^{n} /(n-1, \ldots, 2,1)$.

\subsection{Low valleys on Fuss-Catalan paths}

The notion of Dyck paths can be generalized to Fuss-Catalan paths [11]. For any integer $k \geq 1$, a $k$-Fuss-Catalan path of length $n$ is a lattice path from $(0,0)$ to $(n, k n)$, using east steps $E=(1,0)$ and north steps $N=(0,1)$ and staying weakly below the diagonal $y=k x$. An east step is on level $h$ if it falls on $y=h$, and an low east step is an east step which does not touch the line $y=k x$. Let $\mathcal{C}_{n}^{(k)}$ be the set of $k$-Fuss-Catalan paths of length $n$. It is known the cardinality is $\left|\mathcal{C}_{n}^{(k)}\right|=\frac{1}{k n+1}\left(\begin{array}{c}(k+1) n \\ n\end{array}\right)$, a Fuss-Catalan number.

Let $P \in \mathcal{C}_{n}^{(k)}$. For each low east step of $P$ on level $h$, we associate a weight $x_{i}$ where $i \equiv h(\bmod k)$ and $1 \leq i \leq k$. The weight of $P$, denoted by $w_{e}(P)$, is the monomial defined as the product of all weights of its low east steps. Define

$$
H_{n}^{(k)}(\mathbf{x}):=\sum_{P \in \mathcal{C}_{n}^{(k)}} w_{e}(P)
$$

to be the sum of monomials corresponding to all $k$-Fuss-Catalan paths of length $n$. 
A reduce type $\lambda^{\prime}(P)$ of a Dyck path $P$ is the partition obtained by deleting the part of $\lambda(P)$ containing the first east step. For example, the Dyck path $P=E E N E N N E E E N E E N N N N$ has the reduced type $\lambda^{\prime}(P)=(3,2,1)$. The following result reveals the relation between $H_{n}^{(k)}(\mathbf{x})$ and the reduced type of Dyck paths.

Theorem 1.5 (Armstrong and $\mathrm{Eu}[1]$ ). Let $k$ and $n$ be positive integers. Then

$$
H_{n}^{(k)}(\mathbf{x})=\sum_{P \in \mathcal{D}_{n}} h_{\lambda^{\prime}(P)},
$$

where $h$ is the homogeneous symmetric function on variables $x_{1}, x_{2}, \ldots, x_{k}$.

A valley of a Fuss-Catalan path is an east step followed by a north step. A valley is a low valley if the east step does not touch $y=k x$ and is of level $h$ if the east step falls on $y=h$.

Now we put weights on low valleys instead of low east steps. For each low valley of a $k$-Fuss-Catalan path $P$ on level $h$, we associate a weight $x_{i}$ where $i \equiv h(\bmod k)$ and $1 \leq i \leq k$, and the weight of $P$, denoted by $w_{v}(P)$, is the monomial defined by the product of all its weights of the low valleys. Define

$$
E_{n}^{(k)}(\mathbf{x}):=\sum_{P \in \mathcal{C}_{n}^{(k)}} w_{v}(P)
$$

to be the sum of monomials corresponding to all $k$-Fuss-Catalan paths of length $n$.

Our third main result shows that $E_{n+1}^{(k)}(\mathbf{x})$ is a symmetric function and, if expanded in elementary symmetric functions, has indices running over types of all the large Schröder paths of length $n$. This is the Schröder version of Theorem 1.5.

Theorem 1.6 (Theorem 5.1). Let $k$ and $n$ be positive integers. Then

$$
E_{n+1}^{(k)}(\mathbf{x})=\sum_{P \in \mathcal{R}_{n}} e_{\lambda(P)}
$$

where $e$ is the elementary symmetric function on variables $x_{1}, x_{2}, \ldots, x_{k}$.

The rest of the paper is organized as follows. In Section 2 we will introduce the structure "sparse noncrossing partitions" as a key tool, which is the counterpart of noncrossing partitions used for proving results associated to Dyck paths. The proofs of Theorem 1.2, Theorem 1.4, and Theorem 1.6 are put in Section 3, Section 4, and Section 5, respectively.

\section{Schröder paths and sparse noncrossing partitions}

In this sections, we define sparse noncrossing partitions and give a bijection between Schröder paths and sparse noncrossing partitions which preserves their types. 
A partition $P$ of a set $[n]$ is a collection $B_{1}, B_{2}, \ldots, B_{\ell}$ of nonempty disjoint subsets of $[n]$ whose union is $[n] . \quad B_{i}$ 's are called blocks. If $B_{1}, B_{2}, \ldots, B_{\ell}$ are listed in the increasing order of their least elements, the partition $P$ can be written in the canonical sequential form $P=a_{1} a_{2} \cdots a_{n}$ where $i \in B_{a_{i}}$. The type of a noncrossing partition is the integer partition given by its block sizes. For a noncrossing partition $P=(\{1,7,9\},\{2\},\{3\},\{4,6\},\{5\},\{8\})$, its canonical sequential form is 123454161 and its type is $(3,2,1,1,1,1)$.

The standard representation of a partition $P$ is a graph on the vertex set $[n]$ such that a block $B=\left\{b_{1}, b_{2}, \ldots, b_{k}\right\}$ corresponds to a path $\left(b_{1}, b_{2}, \ldots, b_{k}\right)$. A pair $(i, j)$ with $i<j$ which corresponds to an edge in the standard representation of $P$ is called an arc. An arc $(i, j)$ is called a nesting arc if there is another $\operatorname{arc}(m, n)$ such that $i<m<n<j$. The arc type of a partition $P$ is the integer partition given by length of paths in the standard representation of $P$. If the type of a partition $P$ is $\left(\lambda_{1}, \lambda_{2}, \ldots, \lambda_{\ell}, 1, \ldots, 1\right)$ with $\lambda_{\ell} \geq 2$, then its arc type is $\left(\lambda_{1}-1, \lambda_{2}-1, \ldots, \lambda_{\ell}-1\right)$.

A set partition is called sparse if no two consecutive integers are in the same block. A partition is noncrossing if $a, c \in B_{i}$ and $b, d \in B_{j}$ for no elements $a<b<c<d$ and $i \neq j$. The partition $P$ listed above is sparse and noncrossing.

Proposition 2.1. There is a bijection between the set of all Schröder paths of length $n$ with type $\lambda$ and the set of all sparse noncrossing partitions of $[n+|\lambda|+1]$ with arc type $\lambda$.

Proof. We trace a Schröder path from left to right and begin with the sequence 1. For $k$ consecutive east steps of height $j-1$,

(1) replace each number $i(>j)$ with $i+k$, and

(2) replace $j$ with $j, j+1, j, j+2, j, \ldots, j+k, j$.

For a diagonal step of initial height $j-1$,

(1) if the diagonal step is on the main diagonal, add $j+1$ at the end.

(2) if the diagonal step is not on the main diagonal, replace each number $i(>j+1)$ with $i+1$, and replace $j+1$ with $j+1, j+2$.

For a north step, do nothing. Then the resulting sequence gives a noncrossing set partition of $[n+|\lambda|+1]$. An example is shown in Figure 1.

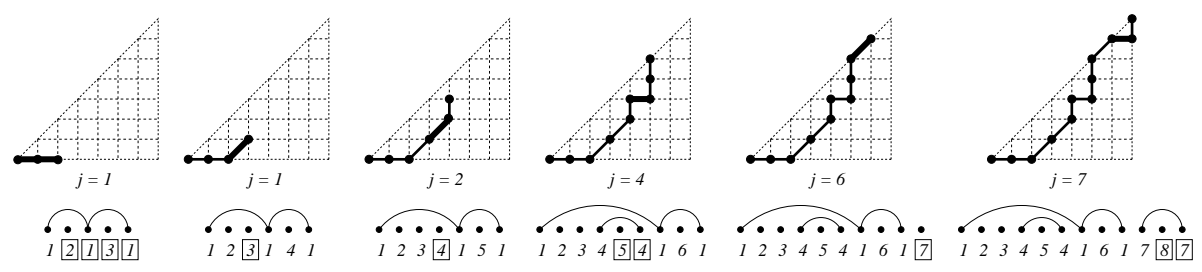

FiguRE 1. From a Schröder path to a sparse noncrossing partition 
Conversely, we first search the numbers which appear more than once in the canonical sequential form of the given noncrossing partition. If $j$ appears $k(>1)$ times, put $k-1$ consecutive east steps on height $j-1$. Next, we search for consecutive pairs $(i, j)$ such that $j$ does not appear before $i$ and $i$ does not appear after $j$. For such a consecutive pair $(i, j)$,

(1) if there is $k$ satisfying $k, \ldots, i, j, \ldots, k$, then put a diagonal step with beginning height $i-2$ between the rightmost east step whose height is lower than $i-1$ and the leftmost east step whose height is higher than $i-2$. If it is necessary, we shift east steps to the right.

(2) if there is no such $k$, then put a diagonal step with beginning height $j-2$ in the same way as above.

Finally, we insert north steps in appropriate positions. Figure 2 shows an example.

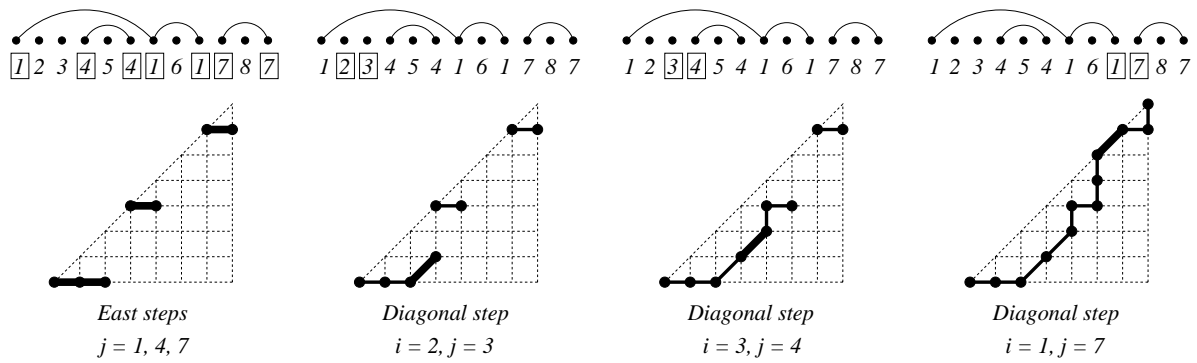

Figure 2. From a sparse noncrossing partition to a Schröder path

\section{Enumeration of Schröder family by type}

In this section, we give an explicit formula for the number of Schröder paths of given length and type.

The following lemma counts the number of noncrossing partitions without singleton blocks with given arc type.

Lemma 3.1. The number of noncrossing partitions of $[|\lambda|+\ell]$ with arc type $\lambda=\left(\lambda_{1}, \ldots, \lambda_{\ell}\right)$ and $k$ arcs having nesting is

$$
\left(\begin{array}{c}
|\lambda| \\
k
\end{array}\right)\left(\begin{array}{c}
\ell \\
k+1
\end{array}\right) \frac{(\ell-1) !}{m_{\lambda}} .
$$

Proof. First, we assume that every part of $\lambda$ is distinct. Let $B_{1}, \ldots, B_{\ell}$ are the blocks with $\left|B_{i}\right|=\lambda_{i}+1$ and $\alpha_{i 1}, \alpha_{i 2}, \ldots, \alpha_{i \lambda_{i}}$ be the arcs in the block $B_{i}$ from left to right. For a set $A$ of $k$ arcs, let $N C_{A}$ be the set of noncrossing partitions such that precisely the arcs in $A$ have nesting. Let $A_{0}=\left\{\alpha_{11}, \alpha_{21}, \ldots, \alpha_{k 1}\right\}$. We claim that there is a one-to-one correspondence between $N C_{A}$ and $N C_{A_{0}}$ for any set $A$ of $k$ arcs. 
Without loss of generality, we may assume that nesting arcs are to the left of nonnesting arcs in the same block. This can be done by successively moving everything under a nesting arc to the leftmost nonnesting arc of the same block.

Let $\beta$ be the rightmost nesting arc contained in the smallest block with more than one nesting arcs and $\gamma$ be the first arc of the largest block without nesting arcs. There are two cases to consider:

Case 1: (when $\gamma$ is not inside $\beta$ ) In this case, we move everything under $\beta$ to under $\gamma$.

Case 2: (when $\gamma$ is inside $\beta$ ) In this case, there is a sequence of arcs between $\beta$ and $\gamma$. Label those arcs so that $\beta_{0}=\beta, \beta_{n}=\gamma$, and $\beta_{j}$ contains $\beta_{j+1}$. Let $C_{j}$ be the block containing $\beta_{j}$ together with all the blocks inside this block except the ones inside $\beta_{j}$. Let $D_{j}$ be the blocks inside $\beta_{j-1}$ and on the left of $C_{j}$ and $E_{j}$ be the blocks inside $\beta_{j-1}$ and on the right of $C_{j}$. Note that $C_{j}, D_{j}$, or $E_{j}$ may consist of more than one block.

Reorder the blocks in the following way:

(a) Put the blocks $C_{0}$ in the innermost position.

(b) Put the blocks $D_{1}$ on the left of the block $C_{0}$ and the blocks $E_{1}$ on the right of $C_{0}$. Then put the blocks $C_{1}$ so that $\beta_{1}$ contains the blocks $D_{1}, C_{0}$, and $E_{1}$.

(c) Suppose we already reorder the blocks $C_{0}, \ldots, C_{j-1}, D_{1}, \ldots$, $D_{j-1}, E_{1}, \ldots, E_{j-1}$. Put the blocks $D_{j}$ on the left of the blocks $C_{j-1}$ and $E_{j}$ on the right of $C_{j-1}$. Then put the blocks $C_{j}$ so that $\beta_{j}$ contains the blocks $C_{0}, \ldots, C_{j-1}, D_{1}, \ldots, D_{j}, E_{1}, \ldots, E_{j}$.

(d) Put all the remaining blocks in the same position as they were. After finishing the reordering, the order of arc (from the outside) is $\beta_{n}, \ldots, \beta_{1}, \beta_{0}$. Moreover, the arc $\beta$ does not have the nesting while the arc $\gamma$ has a nesting.

After finishing this algorithm, we get a noncrossing partition whose set of nesting arcs is $(A \backslash\{\beta\}) \cup\{\gamma\}$. Continuing this algorithm provides a noncrossing partition in $N C_{A_{0}}$. As this algorithm can be easily reversed, there is a one-toone correspondence between $N C_{A}$ and $N C_{A_{0}}$.

Replacing left endpoints of each $a_{i 1}$ with left parentheses and right endpoints of $a_{i 1}$ with right parentheses and removing all the other arcs will give an expression containing $\ell$ pairs of matching parentheses and exactly $\ell-k$ pairs of consecutive parentheses (). Since the number of such expressions is the Narayana number $\frac{1}{\ell}\left(\begin{array}{c}\ell \\ \ell-k\end{array}\right)\left(\begin{array}{c}\ell \\ \ell-k-1\end{array}\right)$ (see [3]) and $k !(\ell-k)$ ! noncrossing partitions in $N C_{A_{0}}$ give the same expressions, there are

$$
\frac{1}{\ell}\left(\begin{array}{c}
\ell \\
\ell-k
\end{array}\right)\left(\begin{array}{c}
\ell \\
\ell-k-1
\end{array}\right) k !(\ell-k) !=\left(\begin{array}{c}
\ell \\
k+1
\end{array}\right)(\ell-1) !
$$

noncrossing partitions in $N C_{A_{0}}$.

Since there are $\left(\begin{array}{c}|\lambda| \\ k\end{array}\right)$ ways to choose $k$ arcs which have nesting, the number of noncrossing partitions of $[|\lambda|+\ell]$ with arc type $\lambda$ when every part of $\lambda$ is 


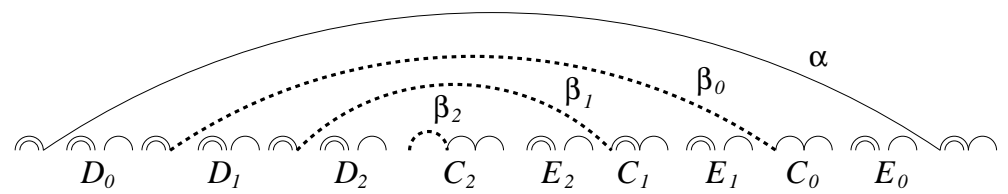

(Before)

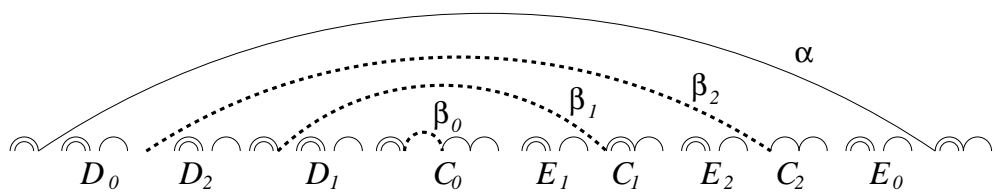

(After)

FIGURE 3. Before and after reordering the blocks when $\gamma=\beta_{2}$ is inside $\beta=\beta_{0}$

distinct is

$$
\left(\begin{array}{c}
|\lambda| \\
k
\end{array}\right)\left(\begin{array}{c}
\ell \\
k+1
\end{array}\right)(\ell-1) !
$$

Because interchanging the elements of the blocks of the same size does not lead to a different partition, we must divide by $m_{\lambda}$ to get the desired result.

Theorem 3.2. The number of all Schröder paths of length $n$ with type $\lambda=$ $\left(\lambda_{1}, \ldots, \lambda_{\ell}\right)$ is

$$
\frac{(\ell-1) !}{m_{\lambda}}\left(\begin{array}{c}
n+1 \\
|\lambda|+1
\end{array}\right)\left(\begin{array}{c}
n \\
\ell-1
\end{array}\right)=\left(\begin{array}{c}
n+1 \\
|\lambda|+1
\end{array}\right) \frac{n(n-1) \cdots(n-\ell+2)}{m_{\lambda}} .
$$

Proof. By Proposition 2.1, $S \operatorname{ch}(n, \lambda)$ is the same as the number of all sparse noncrossing partitions of $[n+|\lambda|+1]$ with arc type $\lambda=\left(\lambda_{1}, \lambda_{2}, \ldots, \lambda_{\ell}\right)$. Note that the number of singleton blocks of noncrossing partitions of $[n+|\lambda|+1]$ with arc type $\lambda$ is $n-\ell+1$. Lemma 3.1 implies that there are

$$
\left(\begin{array}{c}
|\lambda| \\
k
\end{array}\right)\left(\begin{array}{c}
\ell \\
k+1
\end{array}\right) \frac{(\ell-1) !}{m_{\lambda}}
$$

noncrossing partitions of $[|\lambda|+\ell]$ with arc type $\lambda$ with $k$ arcs having nesting. In order to get a sparse set partitions, we first insert one singleton block under each of $|\lambda|-k$ arcs without nesting. Then there are $\left(\left(\begin{array}{c}|\lambda|+\ell+1 \\ n-\ell+1-(|\lambda|-k)\end{array}\right)\right)$ ways to insert remaining singleton blocks to get a sparse noncrossing set partition of 
$[n+|\lambda|+1]$ with arc type $\lambda$. Therefore we have

$$
\begin{aligned}
\operatorname{Sch}(n, \lambda) & =\sum_{k=0}^{\ell-1}\left(\begin{array}{c}
|\lambda| \\
k
\end{array}\right)\left(\begin{array}{c}
\ell \\
k+1
\end{array}\right) \frac{(\ell-1) !}{m_{\lambda}}\left(\left(\begin{array}{c}
|\lambda|+\ell+1 \\
n-\ell+1-(|\lambda|-k)
\end{array}\right)\right) \\
& =\frac{(\ell-1) !}{m_{\lambda}} \sum_{k=0}^{\ell-1}\left(\begin{array}{c}
|\lambda| \\
k
\end{array}\right)\left(\begin{array}{c}
\ell \\
k+1
\end{array}\right)\left(\begin{array}{c}
n+1+k \\
|\lambda|+\ell
\end{array}\right) \\
& =\frac{(\ell-1) !}{m_{\lambda}}\left(\begin{array}{c}
n+1 \\
|\lambda|+1
\end{array}\right)\left(\begin{array}{c}
n \\
\ell-1
\end{array}\right) .
\end{aligned}
$$

The last equality is obtained from Nanjundiah's form of Saalschutz's theorem [10].

For $\lambda \vdash n$, Theorem 3.2 implies Theorem 1.1.

Corollary 3.3. The number of sparse noncrossing partitions of $[n]$ with type $\mu=\left(\mu_{1}, \ldots, \mu_{\ell}\right)$ is

$$
\frac{(\ell-1) !}{m_{\mu}}\left(\begin{array}{c}
\ell \\
n-\ell+1
\end{array}\right) .
$$

Proof. If $m_{1}(\mu)=k$, then the corresponding arc type is $\bar{\mu}=\left(\mu_{1}-1, \mu_{2}-\right.$ $\left.1, \ldots, \mu_{\ell-k}-1\right)$ and the length of the corresponding Schröder path is $\ell-1$. Since $m_{\bar{\mu}}=m_{\mu} / k$ !, the result follows from Theorem 3.2.

\section{Partial horizontal strips and elementary symmetric functions}

In this section, we give results related to elementary symmetric functions.

In order to prove Theorem 1.4, we give the following which describes the relationship between partial horizontal strips and Schröder paths.

Proposition 4.1. There is a bijection from the set of all Schröder paths of length $n$ to the set of all partial horizontal strips in the skew shape $n^{n} /(n-$ $1, \ldots, 2,1)$ which preserves types.

Proof. We trace a Schröder path from left to right. When there is an east step $(i-1, j-1) \rightarrow(i, j-1)$, we put a box on $j$ th row of $i$ th column. When there is a diagonal step $(i, j) \rightarrow(i+1, j+1)$, we take $i-j$ columns of rightmost chosen boxes and shift them to the right by one.

Conversely, we trace a partial horizontal strip from right to left. When there is a box on $j$ th row of $i$ th column, we put an east step $(i-1, j-1) \rightarrow(i, j-1)$. When there is no box on $i$ th column, count how many east steps below $i$ th row. If there are $k$ east steps below $i$ th row, put a diagonal step $(i-1, i+k-1) \rightarrow$ $(i, i+k)$ and shift east steps below $i$ th row to the left.

The following theorem immediately follows from Proposition 3.2 and Proposition 4.1 . 


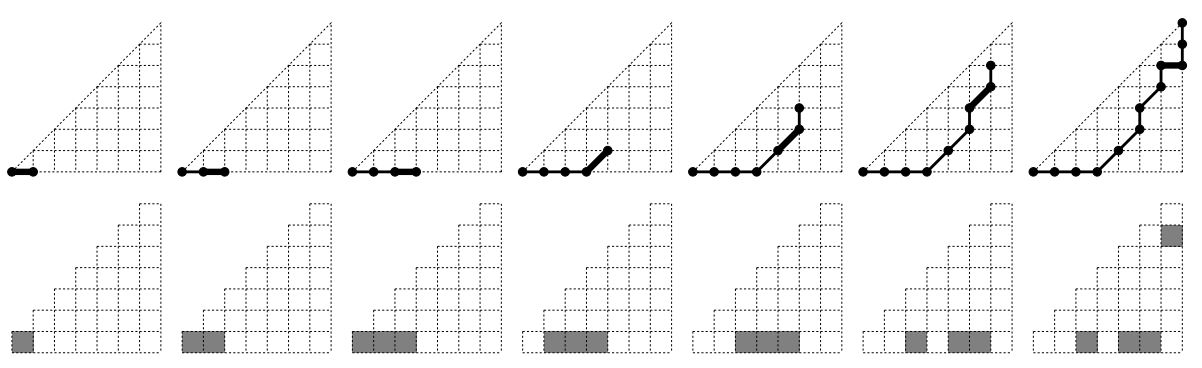

FiguRE 4. From a Schröder path to a partial horizontal strip

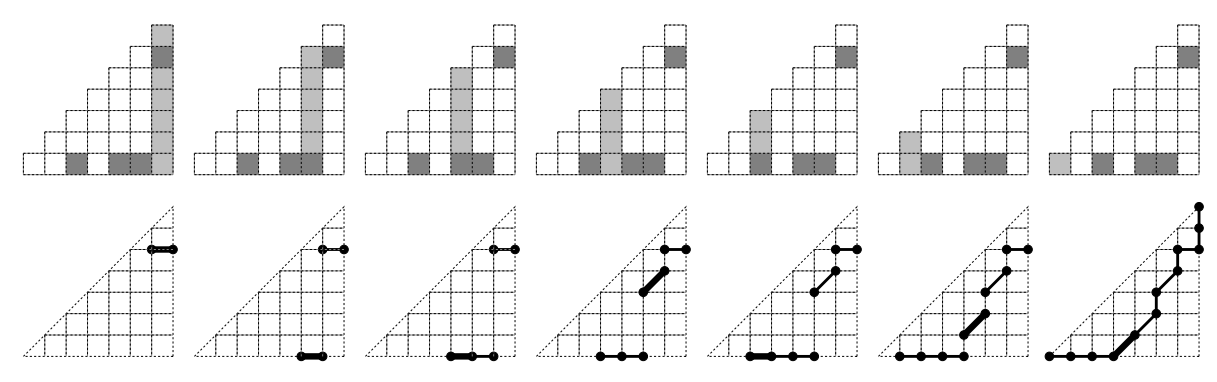

FIGURE 5. From a partial horizontal strip to a Schröder path

Theorem 4.2. We have

$$
\sum_{\sigma} e_{\sigma}=\sum_{\lambda \vdash n}\left(\begin{array}{c}
n+1 \\
|\lambda|+1
\end{array}\right) \frac{n(n-1) \cdots(n-\ell+2)}{m_{\lambda}} e_{\lambda},
$$

where the sum on the left is over all partial horizontal strips $\sigma$ contained in the staircase skew shape $n^{n} /(n-1, \ldots, 2,1)$.

\section{Fuss-Catalan paths and elementary symmetric functions}

This section provides the proof of Theorem 1.6.

Theorem 5.1. Let $k$ and $n$ be positive integers. Then

$$
E_{n+1}^{(k)}(\mathbf{x})=\sum_{P \in \mathcal{R}_{n}} e_{\lambda(P)}
$$

where $e$ is the elementary symmetric function on variables $x_{1}, x_{2}, \ldots, x_{k}$.

Proof. For each $k$-Fuss-Catalan path of length $n$, we associate a partial horizontal strip $\sigma$ in $n^{n} \backslash(n-1, \ldots, 2,1)$ and a monomial in $e_{\lambda(\sigma)}$. Let $P$ be a Fuss-Catalan path in $\mathcal{C}_{n}^{(k)}$. For a low valley beginning at $(i, j k+m)$ with $0 \leq m<k$, we choose a box in $i$ th column and $(j+1)$ st row in the skew 
shape $n^{n} \backslash(n-1, \ldots, 2,1)$. Then the collection of chosen boxes forms a partial horizontal strip $\sigma$ and the weight $w_{v}(P)$ is a monomial in $e_{\lambda(\sigma)}$.

On the other hand, for each partial horizontal strip $\sigma$ and a monomial in $e_{\lambda(P)}$, we associate a $k$-Fuss-Catalan path of length $n$. Let $\sigma$ be a partial horizontal strip in $n^{n} \backslash(n-1, \ldots, 2,1)$ and $m_{1} m_{2} \cdots m_{n}$ be a monomial in $e_{\lambda(P)}$. We may assume that $m_{j}$ is a monomial in $e_{\lambda_{j}}$ where $\lambda_{j}$ is the number of boxes in the $j$ th row of $\sigma$. If $m_{j}=x_{i_{1}} \cdots x_{i_{j}}$, then put valleys in the level $(j-1) k+i_{1}, \ldots,(j-1) k+i_{j}$ whose $x$-coordinates are determined by the position of boxes in the $j$ th row of $\sigma$. Since the positions of low valleys completely determine the Fuss-Catalan paths, we get a Fuss-Catalan path $P$ in $\mathcal{C}_{n}^{(k)}$ and the weight $w_{v}(P)$ is $m_{1} m_{2} \cdots m_{n}$.

Acknowledgments. Authors would like to thank the referee for a careful reading of the manuscript, and valuable suggestions for improvement.

\section{References}

[1] D. Armstrong and S.-P. Eu, Nonhomogeneous parking functions and noncrossing partitions, Electron. J. Combin. 15 (2008), no. 1, Research Paper 146, 12 pp.

[2] J. Bandlow, E. S. Egge, and K. Killpatrick, A weight-preserving bijection between Schröder paths and Schröder permutations, Ann. Comb. 6 (2002), no. 3-4, 235-248.

[3] N. Dershowitz and S. Zaks, Enumerations of ordered trees, Discrete Math. 31 (1980), no. $1,9-28$.

[4] E. S. Egge, J. Haglund, K. Killpatrick, and D. Kremer, A Schröder generalization of Haglund's statistic on Catalan paths, Electron. J. Combin. 10 (2003), Research Paper $16,21 \mathrm{pp}$.

[5] S.-P. Eu and T.-S. Fu, Lattice paths and generalized cluster complexes, J. Combin. Theory Ser. A 115 (2008), no. 7, 1183-1210.

[6] A. M. Garsia and M. Haiman, A remarkable $q, t$-Catalan sequence and $q$-Lagrange inversion, J. Algebraic Combin. 5 (1996), no. 3, 191-244.

[7] J. Haglund, A proof of the q,t-Schröder conjecture, Int. Math. Res. Not. (2004), no. 11, $525-560$.

[8] M. D. Haiman, Conjectures on the quotient ring by diagonal invariants, J. Algebraic Combin. 3 (1994), no. 1. 17-76.

[9] G. Kreweras, Sur les partitions non croisées d'un cycle, Discrete Math. 1 (1972), no. 4, 333-350.

[10] T. S. Nanjundiah, Remark on a note of P. Turán, Amer. Math. Monthly 65 (1958), 354.

[11] J. H. Przytycki and A. S. Sikora, Polygon dissections and Euler, Fuss, Kirkman, and Cayley Numbers, J. Combin. Theory Ser. A 92 (2000), no. 1, 68-76.

[12] R. P. Stanley, Parking functions and noncrossing partitions, The Wilf Festschrift (Philadelphia, PA, 1996), Electron. J. Combin. 4 (1997), no. 1, Research Paper 20, $14 \mathrm{pp}$.

[13] — Enumerative combinatorics. Vol. 2, volume 62 of Cambridge Studies in Advanced Mathematics, Cambridge University Press, Cambridge, 1999.

[14] J. West, Generating trees and the Catalan and Schröder numbers, Discrete Math. 146 (1995), no. 1-3, 247-262. 
Su HyUNG AN

Department of Mathematics

YONSEI UNIVERSITY

Seoul 120-749, Korea

E-mail address: hera1973@yonsei.ac.kr

Sen-Peng Eu

Department of Mathematics

National Taiwan Normal University

TAIPEI 116, TAIWAN, ROC

E-mail address: speu@ntnu.edu.tw

SANGWOOK KIM

Department of Mathematics

Chonnam National University

GWAnguU 500-757, Korea

E-mail address: swkim.math@chonnam.ac.kr 